\title{
Electromagnetic Field Enhancement on Axially Heterostructured NWs: The Role of the Heterojunctions
}

\author{
J.L. PURA @i, ${ }^{1,4}$ J. SOUTO, ${ }^{1}$ P. PERIWAL,${ }^{2,3}$ T. BARON, ${ }^{2,3}$ and J. JIMÉNEZ ${ }^{1}$ \\ 1.-GdS Optronlab, Departamento de Física de la Materia Condensada, Ed. LUCIA, Universidad \\ de Valladolid, Paseo de Belén 19, 47011 Valladolid, Spain. 2.-University Grenoble Alpes, LTM, \\ 38000 Grenoble, France. 3.-CNRS, LTM, 38000 Grenoble, France. 4.—e-mail: jlpura@fmc.uva.es
}

Semiconductor nanowires are the building blocks of future nanoelectronic devices. The study of the interaction between nanowires and visible light reveals resonances that promise light absorption/scattering engineering for photonic applications. We carried out experimental measurements through the micro-Raman spectroscopy of different group IV nanowires, both homogeneous Si nanowires and axially heterostructured SiGe/Si nanowires. These experimental measurements show an enhancement of the Raman signal in the vicinity of the heterojunction of SiGe/Si nanowires. The results are analysed in terms of the electromagnetic modelling of the light/nanowire interaction using finite element methods. The presence of axial heterostructures is shown to produce electromagnetic resonances, and the results are understood as a consequence of a finite change in the relative permittivity of the material at the SiGe/Si heterojunction. This effect opens a path to controlling interactions between light and matter at the nanoscale with direct applications in photonic nanodevices.

Key words: Nanowires, silicon, light-matter interaction, light enhancement, heterojunctions

\section{INTRODUCTION}

Semiconductor nanowires (NWs) have received a great deal of attention in recent years. One of their main fields of interest concerns photonics, as it has been shown that light couples to NWs in different ways depending on the NW diameter, NW composition, light wavelength, and the dielectric mismatch between the NW and the surrounding media. ${ }^{1}$ As a result, all these variables allow us to tune the optical properties of NWs. One of the most relevant properties concerning the light/NW interaction is the ability of NWs to enhance the optical absorption/scattering for certain diameters. ${ }^{2}$ Up to now the research has been focused on both homogeneous and core-shell NWs, while less attention has been paid to the optical properties of axially heterostructured NWs. We aim to unravel the

(Received November 13, 2017; accepted May 3, 2018; published online May 14, 2018) interesting effects that appear when an axially heterostructured NW interacts with an electromagnetic (EM) field. For this, micro-Raman ( $\mu$-R) spectroscopy is used as a local probe of the EM field. Since the Raman signal is proportional to the excitation light intensity, i.e. $|E|^{2}$, the Raman signal arising from each region of the heterostructured NW provides a measurement of the mean value of the EM field distribution in this specific region. As a result, $\mu$-Raman spectroscopy is a very suitable tool to detect the EM field distribution induced inside heterostructured NWs by an incident laser beam.

\section{EXPERIMENTAL AND SAMPLES}

$\mu$-R spectra were recorded with a Labram UV-HR 800 Raman spectrometer from Horiba-Jobin-Yvon. The excitation and the scattered light collection were performed by means of a confocal metallographic microscope with a high magnification objective (X100) and 0.95 numerical aperture (NA). A 
frequency doubled Nd:YAG laser (532 nm) was used as the excitation source. The laser beam diameter at the focal plane is slightly below $1 \mu \mathrm{m}$ according to the Abbe's formula $(\phi=1.22 \lambda / N A)$, which is several times larger than the diameter of the studied NWs, which typically ranged from $30 \mathrm{~nm}$ to $100 \mathrm{~nm}$. The Raman spectra were recorded at different positions along the NWs, in steps of $100 \mathrm{~nm}$. It is important to note that the effective excitation power on the NW depends on its position inside the Gaussian power distribution of the focused laser beam. $^{3,4}$

Group IV heterostructured NWs were grown by the Au-catalized vapour-liquid-solid (VLS) method using a hot-wall CVD reactor (Easy Tube 3000 First Nano, a division of CVD Equipment Corporation). Either $50 \mathrm{~nm} \mathrm{Au}$ colloid nanoparticles or a $2 \mathrm{~nm}$ thick Au layer were deposited on a Si (111) substrate for the NW growth, $\mathrm{SiH}_{4}$ and $\mathrm{GeH}_{4}$ were used as precursors, and $\mathrm{HCl}$ as carrier gas. In order to obtain a heterostructured NW the carrier gas fluxes were changed during the growth run. For example, during the growth of a SiGe segment the Ge flux was switched-off, then the pure Si segment started to grow forming the $\mathrm{SiGe} / \mathrm{Si}$ heterojunction (HJ), called trailing $\mathrm{HJ}$; the leading $\mathrm{HJ}$ was formed by switching-on the Ge flux. For further details about NW growth see. ${ }^{5,6}$

Prior to the Raman measurements, the dimensions and morphological features of each NW were studied in a high resolution field emission scanning electron microscope (FESEM), Fig. 1a, b and c. The NWs' lengths varied from 2 to $12 \mu \mathrm{m}$, and they appear as straight cylinders without appreciable tapering. Transmission Electron Microscopy (TEM) showed the excellent crystallinity of the NWs, Fig. 1c. In particular, high resolution TEM images of the HJ region of $\mathrm{SiGe} / \mathrm{Si} \mathrm{NWs}$ did not show structural discontinuities at the junction, nor stacking faults or other structural defects. ${ }^{5}$

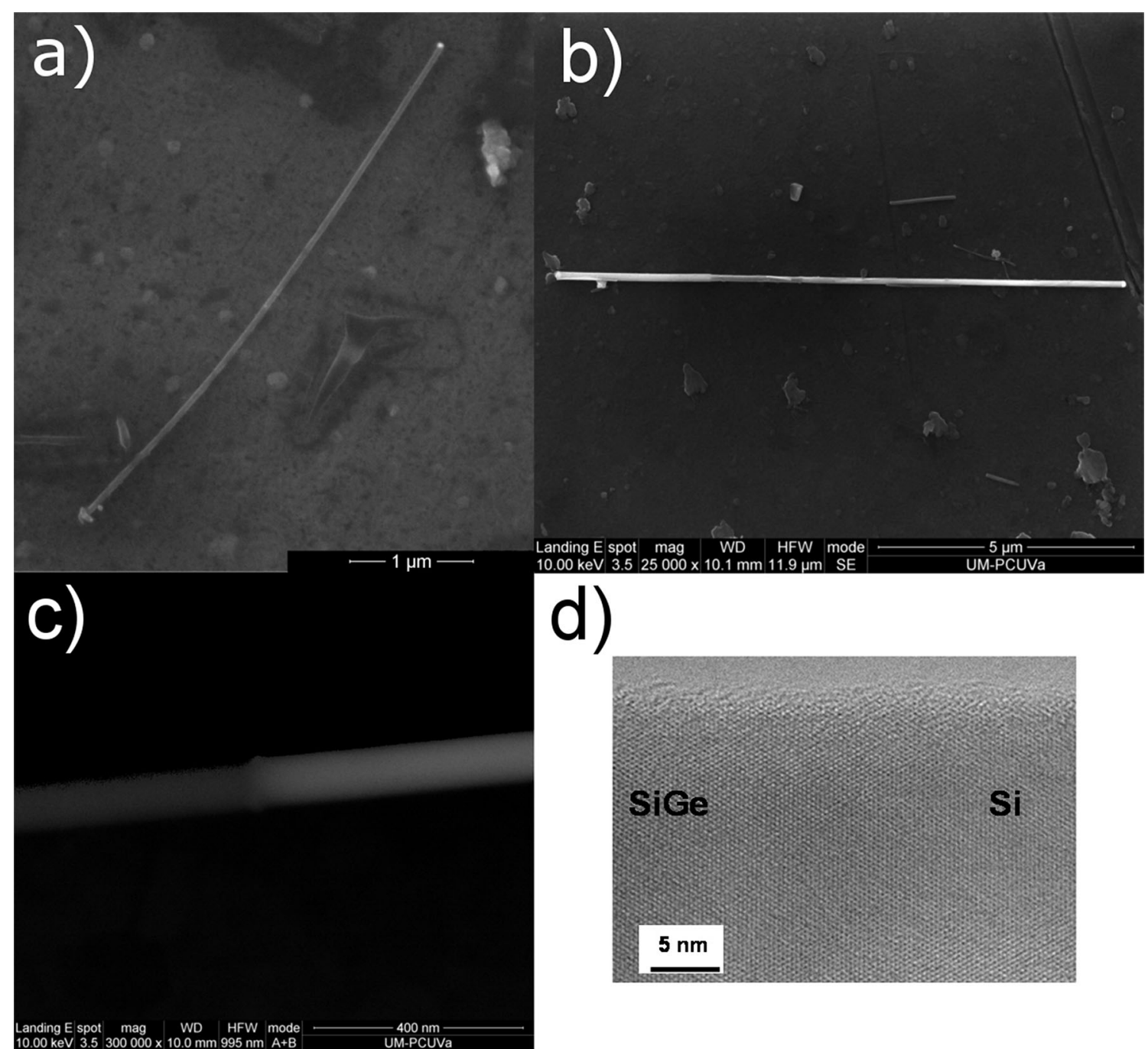

Fig. 1. (a) SEM image of a Si NW (diameter $\approx 30 \mathrm{~nm}$ ). (b) SEM image of a SiGe/Si heterostructured NW (diameter $\approx 100 \mathrm{~nm}$ ). (c) Backscattering SEM image of the HJ region of $\mathrm{a} \approx 107 \mathrm{~nm}$ NW. (d) HR-TEM image of the HJ region of a SiGe/Si axially hetersostructured NW showing the excellent crystalline quality and absence of defects. 
As-grown NWs were separated from the substrate in an ultrasonic bath, and suspended in methanol. Droplets of this suspension were spread out on an $\mathrm{Al}$ coated Si substrate for the $\mu$-R measurements. The choice of the substrates is based on the optimization of the Raman signal, which is enhanced by the presence of a metallic substrate with respect to free standing NWs. Also, but not less important, the presence of the metallic substrate improves the thermal management of the NW, allowing us to minimize laser-induced heating during the $\mu$-R experiments. $\mathrm{Al}$ coated substrates proved to be optimal in order to meet both requirements, so they were used as the standard substrate for the experimental measurements. The Al coating was thick enough to block any optical signal arising from the Si substrate.

\section{EXPERIMENTAL RESULTS}

The longitudinal scans along several heterostructured NWs show a clear enhancement of the Raman signal at the HJ region, see Fig. 2. It can be seen that the signal of the Si-Si (SiGe) Raman mode is greatly enhanced when the laser beam excites the HJ. The Raman shift of this mode corresponds to an intermediate Ge composition between that of $\mathrm{Si}$ and $\mathrm{SiGe}(\sim 60 \% \mathrm{Ge})$ homogeneous segments of the NW, so we deduce that it arises from the transition region, i.e. the HJ. In fact, this Raman band is not detected in the homogeneous segments. Moreover, we have to consider that the Raman signal is proportional to the volume probed by the laser beam in each region of the NW. The transition region between $\mathrm{Si}$ and $\mathrm{SiGe}$ is not an abrupt transition but a gradual change from the material composition of the SiGe segment $(\sim 60 \% \mathrm{Ge})$ to the

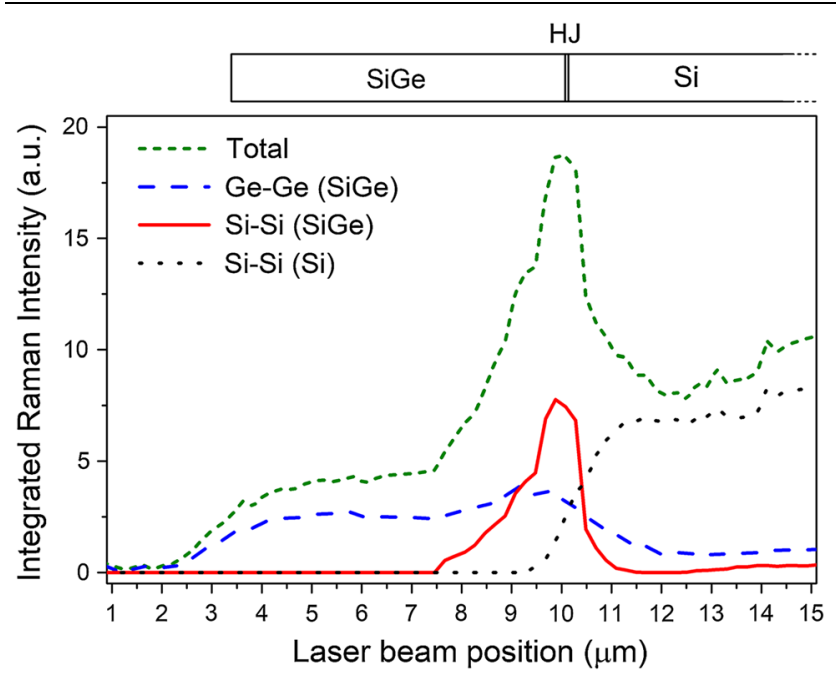

Fig. 2. Raman intensity profiles along a SiGe/Si heterostructured NW showing the enhancement of the signal arising from the HJ. Two Raman modes of the $\mathrm{Si}_{1-x} \mathrm{Ge}_{x}$ alloy are represented, $\mathrm{Ge}-\mathrm{Ge}$ (SiGe) and $\mathrm{Si}-\mathrm{Si}(\mathrm{SiGe})$, as well as the Raman signal arising from pure $\mathrm{Si}, \mathrm{Si}-$ $\mathrm{Si}(\mathrm{Si})$ and the total Raman signal. pure Si segment as a consequence of the reservoir effect of the catalyst droplet. ${ }^{5,7}$ According to the reservoir effect, the HJ extension ranges in the order of magnitude of the NW diameter, so it can be estimated to be around $50 \mathrm{~nm}$ or less. Meanwhile, when the laser beam is focused on the HJ, the homogeneous segments of the NW will be sharing the beam spot with the HJ in a proportion $\approx 500: 50: 500$. According to this estimation the scattering volume ratio between the $\mathrm{HJ}$ and the homogeneous segments is approximately 1 to 10 , i.e. the HJ volume is 10 times smaller than each of the illuminated homogeneous segments. However, we detect a Raman signal arising from the HJ even higher than that arising from the $\mathrm{Si}$ and SiGe segments. Therefore, the Raman signal that originates at the HJ is enhanced by a factor of at least 10 times with respect to a homogeneous NW of the same diameter. A more detailed calculation with the experimental data of these specific $\mathrm{SiGe} / \mathrm{Si}$ heterostructured NWs gives an experimental enhancement factor of circa 63. The enhancement effect in these NWs with a greater Ge composition $(\sim 60 \% \mathrm{Ge})$ is more intense than that observed in NWs with lower Ge composition. ${ }^{8}$ Moreover, due to the higher Ge composition of the NWs, the Raman bands are clearly separated and there is no need for a deconvolution of the Raman spectra.

\section{FINITE ELEMENT METHODS SIMULATIONS}

Parallel to the Raman spectroscopy measurements, the light/NW interaction was studied by means of Finite Element Methods (FEM) simulations. For this the "Electromagnetic Waves in

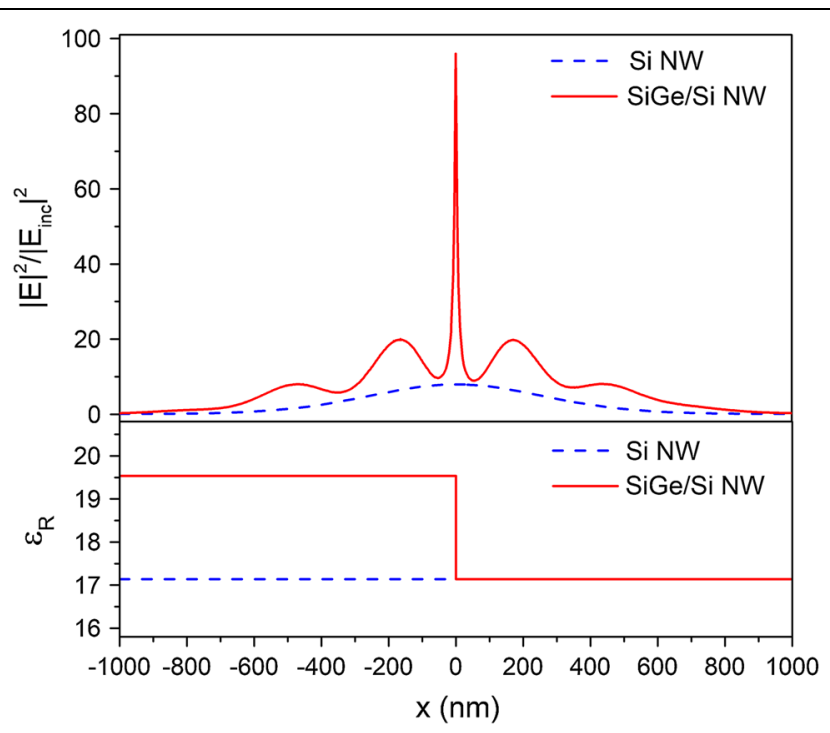

Fig. 3. (Top) EM field distribution inside a homogeneous Si NWs, and a SiGe/Si heterostructured NW. (Bottom) relative electric permittivity along the heterostructured NW. The presence of a discontinuity on the relative permittivity in the heterostructured NW induces the enhancement of the incident field near the HJ region as compared to the homogeneous NWs. 


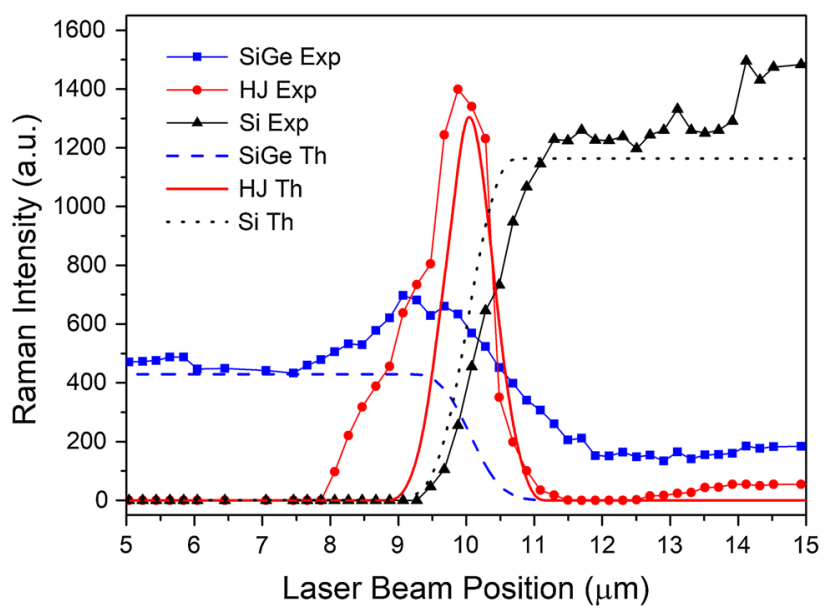

Fig. 4. Simulation of the longitudinal Raman profile measurements along a heterostructured SiGe/Si NW. The results of the simulation are represented by lines and compared with the experimental data of Fig. 2, plotted with symbols. The simulations are in good agreement with the experimental Raman measurements.

Frequency Domain" module of COMSOL Multiphysics software was used. In our model a finite heterostructured NW, with characteristics similar to the experimentally studied ones, was located on an aluminium substrate and surrounded by air. The entire system was limited by Cartesian perfectly matched layers (PMLs), and illuminated by a linearly polarized Gaussian laser beam with $532 \mathrm{~nm}$ wavelength and around $1 \mu \mathrm{m}$ spot size, to reproduce the experimental conditions of the Raman spectra. The position of the excitation beam axis could be varied to reproduce the experimental profile measurements. The electromagnetic field distribution inside the HJ NW is calculated as a function of the position of the excitation laser beam. In this situation, the value of the square of the electric field modulus $|E|^{2}$ will be proportional to the local light intensity; as a consequence it will be proportional to the locally generated Raman signal. Then, by integrating $|E|^{2}$ over the different regions of the heterostructured NW for each position of the laser beam, one can calculate the theoretical Raman profile along the NW axis for comparison with the experimental profile.

The FEM results for these NWs are summarized in Figs. 3 and 4. Figure 3 shows the EM field distribution inside the heterostructured NW computed when the laser beam was centred on the HJ region. The EM field distribution is compared with that of a homogeneous Si NW, where there is no change in the relative permittivity, $\varepsilon$. According to this, the EM field distribution inside the Si NW resembles the Gaussian distribution of the incident laser beam. In the case of the heterostructured NW the system has a finite jump discontinuity in its relative permittivity; when Maxwell equations are solved this results in a local enhancement of the EM field distribution right at the HJ. Once the model was defined, the excitation beam position was varied to reproduce the experimental longitudinal scanning measurement. The EM field distribution is computed for each position of the laser beam, then the theoretical Raman intensities of all NW regions can be calculated from the field distribution. In Fig. 4 the theoretical Raman intensity profile is plotted and compared with the experimental measurements of Fig. 2. We can see a very good agreement between both data. Moreover, if we compute the theoretical Raman enhancement of the HJ signal with this model, the calculated value is 60 , quite close to the experimental value of circa 63 . We can conclude that the model accounts for the experimentally observed Raman enhancement and provides an accurate framework for understanding the physics of the light/heterostructured NW interaction.

\section{CONCLUSIONS}

Heterostructured Si/SiGe NWs present an enhancement of the EM field at the HJ transition region. This effect was measured by $\mu$-Raman spectroscopy detecting an enhancement of the Raman signal arising from the HJ up to a factor of 60. Moreover, the experimentally measured longitudinal Raman profiles show a high increase of the total Raman signal when the excitation beam is crossing the HJ region. The experimental results was simulated by a FEM model, in which the Maxwell equations were solved to find the EM field distribution inside the studied NWs. This model has been proved to explain the EM field localization at the HJ region. At the same time the model reproduces very confidently the Raman longitudinal profiles. As a summary, the change in the relative electric permittivity that takes places at the HJ is shown to be responsible for the EM field localization and enhancement at this region. The control of this EM field enhancement effect with the use of HJs opens the path to improving the performance of a great variety of photonic nanodevices by using heterostructured NWs.

\section{ACKNOWLEDGEMENTS}

This work was funded by Junta de Castilla y León (Projects VA293U13, and VA081U16), and Spanish Government (CICYT MAT2010-20441-C02 (01 and 02)). J. L. Pura was granted by the FPU programme (Spanish Government FPU14/00916).

\section{REFERENCES}

1. F.J. Lopez, J.K. Hyun, U. Givan, I.S. Kim, A.L. Holsteen, and L.J. Lauhon, Nano Lett. 12, 2266 (2012).

2. L. Cao, J.S. White, J.S. Park, J.A. Schuller, B.M. Clemens, and M.L. Brongersma, Nat. Mater. 8, 643 (2009).

3. J. Anaya, A. Torres, A. Martín-Martín, J. Souto, J. Jiménez, A. Rodríguez, and T. Rodríguez, Appl. Phys. A 113, 167 (2013).

4. J. Anaya, A. Torres, A.C. Prieto, V. Hortelano, J. Jiménez, A. Rodríguez, and T. Rodríguez, Appl. Phys. A 114, 1321 (2014). 
5. P. Periwal, N.V. Sibirev, G. Patriarche, B. Salem, F. Bassani, V.G. Dubrovskii, and T. Baron, Nano Lett. 14, 5140 (2014).

6. R.S. Wagner and W.C. Ellis, Appl. Phys. Lett. 4, 89 (1964).
7. T.E. Clark, P. Nimmatoori, K.K. Lew, L. Pan, J.M. Redwing, and E.C. Dickey, Nano Lett. 8, 1246 (2008).

8. J.L. Pura, J. Anaya, J. Souto, C. Prieto, A. Rodríguez, T. Rodríguez, and J. Jiménez, Nanotechnology 27, 455709 (2016). 